Punjab University Journal of Mathematics (2021),53(6),425-434

https://doi.org/1.52280/pujm.2021.530605

\title{
Exact Analytical Solutions of Linear Dissipative Wave Equations via Laplace Transform Method
}

\author{
Muhammad Jamil*, Rahmat Ali Khan and Kamal Shah \\ Department of Mathematics, \\ University of Malakand, Dir(L), Khyber Pakhtunkhwa, Pakistan. \\ Corresponding author Email: jamilkanju@gmail.com
}

Received: 29 November, 2019 / Accepted: 25 June, 2021 / Published online: 25 May, 2021

\begin{abstract}
A wave phenomena evolved day after day, as various concepts regarding waves appeared with the passage of time. These phenomena are generally modelled mathematically by partial differential equations (PDEs). In this research, we investigate the exact analytical solutions of one and two dimensional linear dissipative wave equations which are modelled by second order PDEs with use of some initial and boundary conditions. We use double Laplace transform (DLT) and triple Laplace transform (TLT) methods to determine these exact analytical solutions. We provide examples with figures to test effectiveness of this scheme of Laplace transform.
\end{abstract}

AMS (MOS) Subject Classification Codes: 44A10; 35L05; $35 \mathrm{LS3}$.

Key Words: Double Laplace transform, Triple Laplace Transform, Analytical Solutions, Linear Dissipative Wave Equations.

\section{INTRODUCTION}

The wave equations have many dynamical applications for the characterization of waves in the field of classical physics and engineering. Because, wave equations deal with the important problems of vibrating strings, sound waves and light waves, etc. Moreover, the wave equations are hyperbolic partial differential equations of second order. In general, wave equations concern with time variable $t$ and one or more dimensional variables (spatial variables) $x_{1}, x_{2}, x_{3}, \ldots, x_{n}$. Both linear and non-linear waves equations of second order given in [10], have the general form as

$$
\frac{\partial^{2} \psi}{\partial t^{2}}-\gamma \frac{\partial^{2} \psi}{\partial x^{2}}=g\left(x, t, \psi, \psi^{2}, \alpha \frac{\partial \psi}{\partial t}, \beta \frac{\partial \psi}{\partial x},\left(\frac{\partial \psi}{\partial t}\right)^{2},\left(\frac{\partial \psi}{\partial x}\right)^{2}\right),
$$


where $\psi=\psi(x, t) \in C\left(\mathbb{R}_{+}, \mathbb{R}_{+}\right), \alpha, \beta$ and $\gamma$ are constant coefficients. The assigned initial and boundary conditions are

$$
\begin{gathered}
\text { Initial Conditions (ICs): } \psi(x, 0)=g_{0}(x), \frac{\partial \psi(x, 0)}{\partial t}=g_{1}(x), x \in \mathbb{R}_{+}, \\
\text {Boundary Conditions(BCs): } \psi(0, t)=h_{0}(t), \frac{\partial \psi(0, t)}{\partial x}=h_{1}(t), t \in \mathbb{R}_{+} .
\end{gathered}
$$

Kaya in [10], used the method of Adomian decomposition for solving three different wave equations. In this method numerical approximations of the damped and dissipative wave equations have been derived in a series form. The obtained numerical approximations were correlated with the analytical solutions. Jarfri et al. [6], used an operational matrix with fractional integration based upon linear B-spline function to evaluate solution of fractional partial differential equations. The authors in [7], developed a powerful algorithm based on homogeneous balance method for exact solutions of the fisher equation, telegraph equation, modified equal width equation and Cahn-Allen equation. The developed algorithm was investigated with the support of some relevant examples. Jarfri et al. in [8], compared results obtained by homotopy analysis method applied to evolution equation with Adomian decomposition method and exact solution.

In the field of fractional calculus, some researchers applied a modified variational iteration method (MVIM) with Adomian polynomials to fractional Riccati differential equation [9]. The numerical results obtained by MVIM concluded the best approximations and high convergence. Bokharia et al. in [4], studied applications of shehu transform for AtanganaBalenu fractional derivatives. Korpinar et al. in [12], implemented the method of Laplace homotopy analysis to the fractional model of Fokker-Planck equations.

In this article, we focus at the analytical solutions of one dimensional and two dimensional dissipative wave equations by applying DLT and TLT techniques. Recently, Dhunde and Waghmare in [13], used method of DLT to solve different equations with one dimensional variable $x$ and time variable $t$, which include the class of wellknown Advection Diffusion equation, Reaction Diffusion equation, Telegraph equation, Klein-Gordon equation, Linear Dissipative wave equation, Korteweg-de vries equation and the Euler-Bernoulli equation of Mathematical Physics. The method of Laplace transform (LT) is an essential scheme for acquiring exact solutions of linear differential equations. LT method has many applications in different fields of science and engineering. LT technique converts differential equations into algebraic equations and the convolution into multiplication which easily led one to the solution as it is simple to solve algebraic equations. The main advantage of LT method is that it evaluate exact solutions while the other methods such as variational iteration method and Adomian decomposition method mostly led us to approximate solutions. The method of Laplace transformation has been used intensively for solving various linear equations in many articles, see few of them in $[13,3,1,5,2]$ and the references therein. In [1], Atangana used TLT technique to solve three dimensional partial differential equations.

In this paper, first we evaluate the exact analytical solution of one dimensional (spatial) variable and time dependent linear dissipative wave equation by DLT method, secondly we extend this one dimensional variable and time dependent linear dissipative wave equation to an equation with two spatial variables $x, y$ and time variable $t$ and then evaluate the analytical solution by TLT Method. 
In particular to (1.1), the linear dissipative wave equation dependent of one spatial variable $x$ and time variable $t$ with initial and boundary conditions as in (1.2) and (1.3) is given by

$$
\begin{array}{r}
\alpha \frac{\partial \psi}{\partial t}+\frac{\partial^{2} \psi}{\partial t^{2}}=\beta \frac{\partial \psi}{\partial x}+\gamma \frac{\partial^{2} \psi}{\partial x^{2}}+g(x, t), \quad(x, t) \in \mathbb{R}_{+}^{2}, \\
\text { ICs: } \psi(x, 0)=g_{0}(x), \frac{\partial \psi(x, 0)}{\partial t}=g_{1}(x), x \in \mathbb{R}_{+}, \\
\text {BCs: } \psi(0, t)=h_{0}(t), \frac{\partial \psi(0, t)}{\partial x}=h_{1}(t), t \in \mathbb{R}_{+},
\end{array}
$$

where $\psi=\psi(x, t) \in C\left(\mathbb{R}_{+}, \mathbb{R}_{+}\right), g_{0}, g_{1}, h_{0}, h_{1} \in \mathbb{R}_{+}$and $g(x, t)$ is a source function.

The extension of (1.1) to a linear dissipative wave equation of two spatial variables $x, y$ and time variable $t$ with ICs and BCs is given by

$$
\begin{gathered}
\alpha \frac{\partial \psi}{\partial t}+\frac{\partial^{2} \psi}{\partial t^{2}}=\beta\left[\frac{\partial \psi}{\partial x}+\frac{\partial \psi}{\partial y}\right]+\gamma\left[\frac{\partial^{2} \psi}{\partial x^{2}}+\frac{\partial^{2} \psi}{\partial y^{2}}\right]+g(x, y, t), \quad(x, y, t) \in \mathbb{R}_{+}^{3}, \\
\text { ICs: } \psi(x, y, 0)=g_{0}(x, y), \frac{\partial \psi(x, y, 0)}{\partial t}=g_{1}(x, y), \quad(x, y) \in \mathbb{R}_{+}^{2}, \\
\text { BCs: } \psi(0, y, t)=h_{0}(y, t), \frac{\partial \psi(0, y, t)}{\partial x}=h_{1}(y, t), \quad(y, t) \in \mathbb{R}_{+}^{2}, \\
\psi(x, 0, t)=k_{0}(x, t), \frac{\partial \psi(x, 0, t)}{\partial y}=k_{1}(x, t), \quad(x, t) \in \mathbb{R}_{+}^{2},
\end{gathered}
$$

where $\psi=\psi(x, y, t) \in C\left(\mathbb{R}_{+}^{2}, \mathbb{R}_{+}\right), g_{0}, g_{1}, h_{0}, h_{1}, k_{0}, k_{1} \in C\left(\mathbb{R}_{+}, \mathbb{R}_{+}\right), g(x, y, t)$ is continuous source function. Moreover $\alpha, \beta$ and $\gamma$ are constant coefficients.

\section{PRELIMINARIES}

We add some concerned definitions and lemmas in this section.

Definition 2.1. [14] Let $\psi(x, t)$ be a function of one dimensional variable $x$ and a time variable $t$ defined in positive quadrant of xt-plane. Then the double Lapalace transform of a function $\psi(x, t)$ is given by

$$
L_{x} L_{t}\{\psi(x, t)\}=\bar{\psi}(p, s)=\int_{0}^{\infty} \int_{0}^{\infty} e^{-(p x+s t)} \psi(x, t) d t d x,
$$

where $p$ and $s$ are transform parameters.

Definition 2.2. [15] Let $\psi(x, y, t)$ be a function defined in positive octant of xyt-rectangular coordinate system. Then the triple Lapalace transform of the function $\psi(x, y, t)$ is given by

$$
L_{x} L_{y} L_{t}\{\psi(x, y, t)\}=\bar{\psi}(p, q, s)=\int_{0}^{\infty} \int_{0}^{\infty} \int_{0}^{\infty} e^{-(p x+q y+s t)} \psi(x, y, t) d t d y d x
$$

where $p, q$ and $s$ are transform parameters.

Also we are required the following results as given in [11] of double and triple Laplace transform of partial differential equations, which can be deduce from the definitions 2.1 and 2.2. 
Lemma 2.3. For $\psi=\psi(x, t) \in C\left(\mathbb{R}_{+}, \mathbb{R}_{+}\right)$, the following hold

$$
\begin{aligned}
& L_{x} L_{t}\left\{\frac{\partial^{m} \psi(x, t)}{\partial x^{m}}\right\}=p^{m} \bar{\psi}(p, s)-\sum_{i=0}^{m-1} p^{m-1-i} L_{t}\left\{\frac{\partial^{i} \psi(o, t)}{\partial x^{i}}\right\}, \\
& L_{x} L_{t}\left\{\frac{\partial^{n} \psi(x, t)}{\partial t^{n}}\right\}=s^{n} \bar{\psi}(p, s)-\sum_{j=0}^{n-1} s^{n-1-j} L_{x}\left\{\frac{\partial^{j} \psi(x, 0)}{\partial t^{j}}\right\},
\end{aligned}
$$

where $i=0,1,2, \ldots, m$ and $j=0,1,2, \ldots, n$.

Lemma 2.4. For $\psi=\psi(x, y, t) \in C\left(\mathbb{R}_{+}^{2}, \mathbb{R}_{+}\right)$, the following hold

$$
\begin{gathered}
L_{x} L_{y} L_{t}\left\{\frac{\partial^{l} \psi(x, y, t)}{\partial x^{l}}\right\}=p^{l} \bar{\psi}(p, q, s)-\sum_{i=0}^{l-1} p^{l-1-i} L_{y} L_{t}\left\{\frac{\partial^{i} \psi(0, y, t)}{\partial x^{i}}\right\} \\
L_{x} L_{y} L_{t}\left\{\frac{\partial^{m} \psi(x, y, t)}{\partial y^{m}}\right\}=q^{m} \bar{\psi}(p, q, s)-\sum_{j=0}^{m-1} q^{m-1-j} L_{x} L_{t}\left\{\frac{\partial^{j} \psi(x, 0, t)}{\partial y^{j}}\right\} \\
L_{x} L_{y} L_{t}\left\{\frac{\partial^{n} \psi(x, y, t)}{\partial t^{n}}\right\}=s^{n} \bar{\psi}(p, q, s)-\sum_{k=0}^{n-1} s^{n-1-k} L_{x} L_{y}\left\{\frac{\partial^{k} \psi(x, y, 0)}{\partial t^{k}}\right\}
\end{gathered}
$$

where $i=0,1,2, \ldots, l, j=0,1,2, \ldots, m$ and $k=0,1,2, \ldots, n$.

\section{Analytical Solution of One Dimensional Linear Dissipative Wave}

\section{EQUATION}

Consider the linear dissipative wave equation (1.4) of a time variable $t$ and one spatial variable $x$ and assigning DLT, we have

$$
\alpha L_{x} L_{t} \frac{\partial \psi}{\partial t}+L_{x} L_{t} \frac{\partial^{2} \psi}{\partial t^{2}}=\beta L_{x} L_{t} \frac{\partial \psi}{\partial x}+\gamma L_{x} L_{t} \frac{\partial^{2} \psi}{\partial x^{2}}+L_{x} L_{t} g(x, t),
$$

which by Lemma 2.3 gives

$$
\left\{\begin{array}{l}
\alpha\left\{s \bar{\psi}(p, s)-L_{x} \psi(x, 0)\right\}+\left\{s^{2} \bar{\psi}(p, s)-s L_{x} \psi(x, 0)-L_{x} \frac{\partial \psi(x, 0)}{\partial t}\right\} \\
=\beta\left\{p \bar{\psi}(p, s)-L_{t} \psi(0, t)\right\}+\gamma\left\{p^{2} \bar{\psi}(p, s)-p L_{t} \psi(0, t)-L_{t} \frac{\partial \psi(0, t)}{\partial x}\right\} \\
+L_{x} L_{t} g(x, t) .
\end{array}\right.
$$

Let the laplace transforms of the ICs (1.5) and BCs (1.6) be as follow

$$
\begin{gathered}
L_{x} \psi(x, 0)=\bar{g}_{0}(p), \quad L_{x} \frac{\partial \psi(x, 0)}{\partial t}=\bar{g}_{1}(p), \\
L_{t} \psi(0, t)=\bar{h}_{0}(s), \quad L_{t} \frac{\partial \psi(0, t)}{\partial x}=\bar{h}_{1}(s) .
\end{gathered}
$$

Also let $L_{x} L_{t} g(x, t)=\bar{g}(p, s)$, then equation (3.11) gives

$$
\bar{\psi}(p, s)=\frac{(\alpha+s) \bar{g}_{0}(p)+\bar{g}_{1}(p)-(\beta+\gamma p) \bar{h}_{0}(s)-\gamma \bar{h}_{1}(s)+\bar{g}(p, s)}{s(\alpha+s)-p(\beta+\gamma p)} .
$$


Applying the inverse DLT, gives the analytical solution of (1.4) as

$$
\psi(x, t)=L_{x}^{-1} L_{t}^{-1} \frac{(\alpha+s) \bar{g}_{0}(p)+\bar{g}_{1}(p)-(\beta+\gamma p) \bar{h}_{0}(s)-\gamma \bar{h}_{1}(s)+\bar{g}(p, s)}{s(\alpha+s)-p(\beta+\gamma p)} .
$$

\section{Analytical Solution of Two Dimensional Linear Dissipative Wave EQUATION}

Considering the linear dissipative wave equation (1.7) of a time variable $t$ and two spatial variables $x, y$ and assigning TLT, we have

$\alpha L_{x} L_{y} L_{t} \frac{\partial \psi}{\partial t}+L_{x} L_{y} L_{t} \frac{\partial^{2} \psi}{\partial t^{2}}=\beta L_{x} L_{y} L_{t}\left[\frac{\partial \psi}{\partial x}+\frac{\partial \psi}{\partial y}\right]+\gamma L_{x} L_{y} L_{t}\left[\frac{\partial^{2} \psi}{\partial x^{2}}+\frac{\partial^{2} \psi}{\partial y^{2}}\right]+L_{x} L_{y} L_{t} g(x, y, t)$,

which by Lemma 2.4 gives

$$
\left\{\begin{array}{l}
\alpha\left\{s \bar{\psi}(p, q, s)-L_{x} L_{y} \psi(x, y, 0)\right\} \\
+\left\{s^{2} \bar{\psi}(p, q, s)-s L_{x} L_{y} \psi(x, y, 0)-L_{x} L_{y} \frac{\partial \psi(x, y, 0)}{\partial t}\right\} \\
=\beta\left\{p \bar{\psi}(p, q, s)-L_{y} L_{t} \psi(0, y, t)\right\}+\beta\left\{q \bar{\psi}(p, q, s)-L_{x} L_{t} \psi(x, 0, t)\right\} \\
+\gamma\left\{p^{2} \bar{\psi}(p, q, s)-p L_{y} L_{t} \psi(0, y, t)-L_{y} L_{t} \frac{\partial \psi(0, y, t)}{\partial x}\right\} \\
+\gamma\left\{q^{2} \bar{\psi}(p, q, s)-q L_{x} L_{t} \psi(x, 0, t)-L_{x} L_{t} \frac{\partial \psi(x, 0, t)}{\partial y}\right\} \\
+L_{x} L_{y} L_{t} g(x, y, t) .
\end{array}\right.
$$

Let the double laplace transforms of the ICs (1.8) and BCs (1.9) and (1.10) be as follow

$$
\begin{array}{rlrl}
L_{x} L_{y} \psi(x, y, 0) & =\bar{g}_{0}(p, q), & L_{x} L_{y} \frac{\partial \psi(x, y, 0)}{\partial t}=\bar{g}_{1}(p, q), \\
L_{y} L_{t} \psi(0, y, t) & =\bar{h}_{0}(q, s), & L_{y} L_{t} \frac{\partial \psi(0, y, t)}{\partial x}=\bar{h}_{1}(q, s), \\
L_{x} L_{t} \psi(x, 0, t) & =\bar{k}_{0}(p, s), & L_{x} L_{t} \frac{\partial \psi(x, 0, t)}{\partial y} & =\bar{k}_{1}(p, s) .
\end{array}
$$

Also let $L_{x} L_{y} L_{t} g(x, y, t)=\bar{g}(p, q, s)$, then (4.13) gives

$$
\bar{\psi}(p, q, s)=\frac{(\alpha+s) \bar{g}_{0}+\bar{g}_{1}-(\beta+\gamma p) \bar{h}_{0}-\gamma \bar{h}_{1}-(\beta+\gamma q) \bar{k}_{0}-\gamma \bar{k}_{1}+\bar{g}}{s(\alpha+s)-p(\beta+\gamma p)-q(\beta+\gamma q)} .
$$

Applying the inverse TLT, gives the analytical solution of (1.7) as

$\psi(x, y, t)=L_{x}^{-1} L_{y}^{-1} L_{t}^{-1} \frac{(\alpha+s) \bar{g}_{0}+\bar{g}_{1}-(\beta+\gamma p) \bar{h}_{0}-\gamma \bar{h}_{1}-(\beta+\gamma q) \bar{k}_{0}-\gamma \bar{k}_{1}+\bar{g}}{s(\alpha+s)-p(\beta+\gamma p)-q(\beta+\gamma q)}$.

\section{EXAMPLES}

In this section of examples, we provide some test problems to demonstrate the results established in last two sections. 
Example 5.1. For $\alpha=\beta=\gamma=1$ and $g(x, t)=-3\left(x^{2}+t^{2}\right)-6(x+t)$, we consider the one dimensional linear dissipative wave equation (1.4) with ICs and BCs as

$$
\begin{gathered}
\frac{\partial \psi}{\partial t}+\frac{\partial^{2} \psi}{\partial t^{2}}=\frac{\partial \psi}{\partial x}+\frac{\partial^{2} \psi}{\partial x^{2}}-3\left(x^{2}+t^{2}\right)-6(x+t), \quad(x, t) \in \mathbb{R}_{+}^{2} \\
\text { ICs: } \psi(x, 0)=x^{3}=g_{0}(x), \quad \frac{\partial \psi(x, 0)}{\partial t}=0=g_{1}(x), x \in \mathbb{R}_{+} \\
\text {BCs: } \psi(0, t)=t^{3}=h_{0}(t), \quad \frac{\partial \psi(0, t)}{\partial x}=0=h_{1}(t), t \in \mathbb{R}_{+}
\end{gathered}
$$

where $\psi=\psi(x, t) \in C\left(\mathbb{R}_{+}, \mathbb{R}_{+}\right), g_{0}, g_{1}, h_{0}, h_{1} \in \mathbb{R}_{+}$and $-3\left(x^{2}+t^{2}\right)-6(x+t)$ is $a$ source term. Now substituting

$$
\bar{g}_{0}(p)=\frac{6}{p^{4}}, \quad \bar{g}_{1}(p)=0, \quad \bar{h}_{0}(s)=\frac{6}{s^{4}}, \quad \bar{h}_{1}(s)=0
$$

and $\bar{g}(p, s)=-6\left(\frac{1}{P^{3} s}+\frac{1}{p s^{3}}+\frac{1}{P^{2} s}+\frac{1}{p s^{2}}\right)$ in (3.12), we have the analytical solution of (5.15) as

$\psi(x, t)=L_{x}^{-1} L_{t}^{-1} \frac{1}{s(1+s)-p(1+p)}\left[(1+s) \frac{6}{p^{4}}-(1+p) \frac{6}{s^{4}}-6\left(\frac{1}{p^{3} s}+\frac{1}{p s^{3}}+\frac{1}{p^{2} s}+\frac{1}{p s^{2}}\right)\right]$.

From which we can set $\psi(x, t)=L_{x}^{-1} L_{t}^{-1}\left[\frac{3 !}{p^{4} s}-\frac{3 !}{p s^{4}}\right]$, which gives the exact solution as

$$
\psi(x, t)=x^{3}-t^{3} .
$$

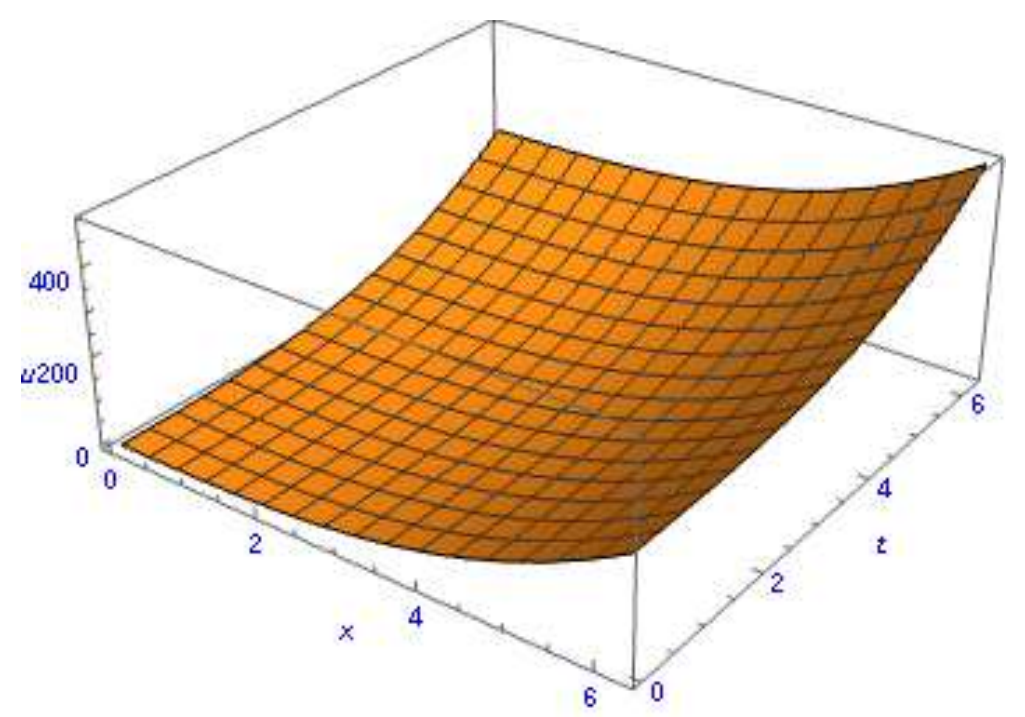

FIGURE 1. Plot for the solution $\psi(x, t)=x^{3}-t^{3}$. 
Example 5.2. For $\alpha=\beta=-1, \gamma=1$ and $g(x, t)=0$, we consider another test problem of one dimensional linear dissipative wave equation (1.4) with ICs and BCs as

$$
\frac{\partial^{2} \psi}{\partial t^{2}}-\frac{\partial \psi}{\partial t}=\frac{\partial^{2} \psi}{\partial x^{2}}-\frac{\partial \psi}{\partial x},(x, t) \in \mathbb{R}_{+}^{2},
$$

ICs: $\psi(x, 0)=\sin x=g_{0}(x), \frac{\partial \psi(x, 0)}{\partial t}=\cos x=g_{1}(x), x \in \mathbb{R}_{+}$,

$$
\text { BCs: } \psi(0, t)=\sin t=h_{0}(t), \quad \frac{\partial \psi(0, t)}{\partial x}=0=h_{1}(t), t \in \mathbb{R}_{+},
$$

where $\psi=\psi(x, t) \in C\left(\mathbb{R}_{+}, \mathbb{R}_{+}\right), g_{0}, g_{1}, h_{0}, h_{1} \in \mathbb{R}_{+}$. Now substituting

$$
\bar{g}_{0}(p)=\frac{1}{1+p^{2}}, \quad \bar{g}_{1}(p)=\frac{p}{1+p^{2}}, \quad \bar{h}_{0}(s)=\frac{1}{1+s^{2}}, \quad \bar{h}_{1}(s)=0,
$$

and $\bar{g}(p, s)=0$ in (3.12), we have the analytical solution of (5.18) as

$$
\psi(x, t)=L_{x}^{-1} L_{t}^{-1} \frac{1}{s(s-1)-p(p-1)}\left[\frac{s-1}{1+p^{2}}+\frac{p}{1+p^{2}}-\frac{p-1}{1+s^{2}}\right],
$$

on simplification we have

$$
\psi(x, t)=L_{x}^{-1} L_{t}^{-1}\left[\left(\frac{1}{1+p^{2}}\right)\left(\frac{s}{1+s^{2}}\right)+\left(\frac{p}{1+p^{2}}\right)\left(\frac{1}{1+s^{2}}\right)\right],
$$

and thus the solution is as below

$$
\psi(x, t)=\sin (x+t) .
$$

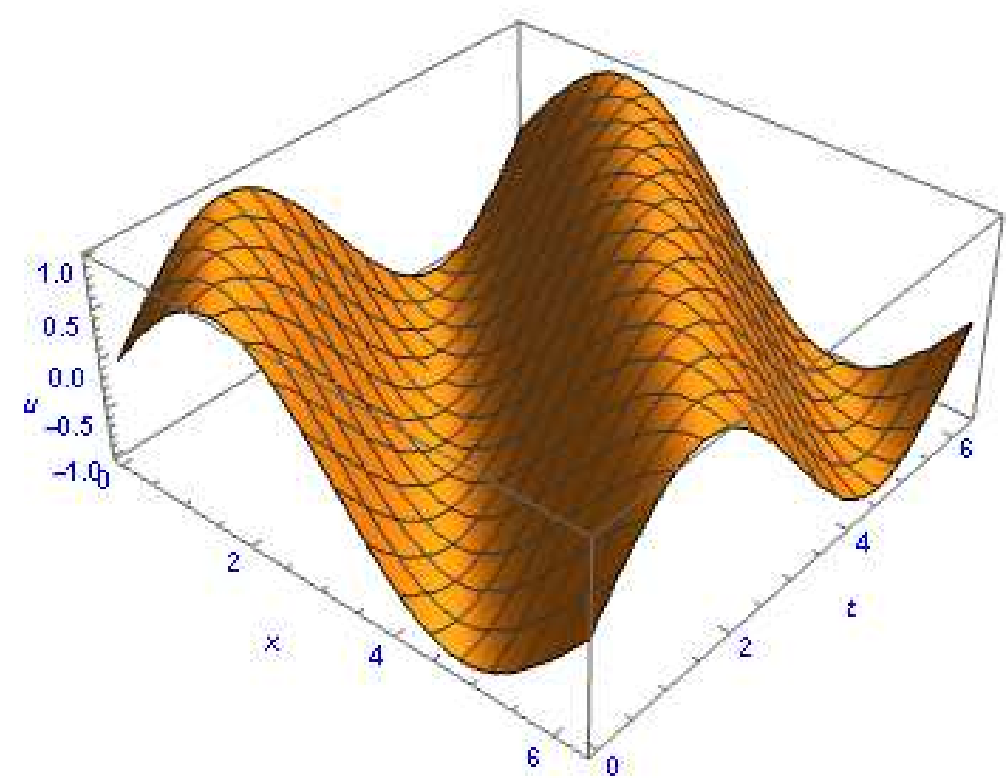

Figure 2. Plot for the solution $\psi(x, t)=\sin (x+t)$. 
Example 5.3. For $\alpha=\beta=\gamma=1$ and $g(x, y, t)=(2 t+1) \cos (x+y)+2 t \sin (x+y)$, we consider the two dimensional linear dissipative wave equation (1.7) with initial and boundary conditions as

$$
\begin{array}{r}
\frac{\partial \psi}{\partial t}+\frac{\partial^{2} \psi}{\partial t^{2}}=\frac{\partial \psi}{\partial x}+\frac{\partial \psi}{\partial y}+\frac{\partial^{2} \psi}{\partial x^{2}}+\frac{\partial^{2} \psi}{\partial y^{2}}+(2 t+1) \cos (x+y)+2 t \sin (x+y), \quad(x, y, t) \in \mathbb{R}_{+}^{3}, \\
\text { ICs: } \psi(x, y, 0)=0=g_{0}(x, y), \quad \frac{\partial \psi(x, y, 0)}{\partial t}=\cos (x+y)=g_{1}(x, y), \quad(x, y) \in \mathbb{R}_{+}^{2}, \\
\text { BCs: } \psi(0, y, t)=t \cos y=h_{0}(y, t), \quad \frac{\partial \psi(0, y, t)}{\partial x}=-t \sin y=h_{1}(y, t), \quad(y, t) \in \mathbb{R}_{+}^{2}, \\
\psi(x, 0, t)=t \cos x=k_{0}(x, t), \quad \frac{\partial \psi(x, 0, t)}{\partial y}=-t \sin x=k_{1}(x, t), \quad(x, t) \in \mathbb{R}_{+}^{2},
\end{array}
$$

where $\psi=\psi(x, y, t) \in C\left(\mathbb{R}_{+}^{2}, \mathbb{R}_{+}\right), g_{0}, g_{1}, h_{0}, h_{1}, k_{0}, k_{1} \in \mathbb{R}_{+}^{2}$. Now substituting

$$
\begin{aligned}
& \bar{g}_{0}(p, q)=0, \quad \bar{g}_{1}(p, q)=\frac{p q-1}{\left(1+p^{2}\right)\left(1+q^{2}\right)}, \quad \bar{h}_{0}(q, s)=\frac{q}{s^{2}\left(1+q^{2}\right)}, \\
& \bar{h}_{1}(s)=\frac{-1}{s^{2}\left(1+q^{2}\right)}, \quad \bar{k}_{0}(p, s)=\frac{p}{s^{2}\left(1+p^{2}\right)}, \quad \bar{k}_{1}(p, s)=\frac{-1}{s^{2}\left(1+p^{2}\right)} \\
& \text { and } \bar{g}(p, q, s)=\left[\frac{2}{s^{2}}+\frac{1}{s}\right] \frac{p q-1}{\left(1+p^{2}\right)\left(1+q^{2}\right)}+\frac{2(p+q)}{s^{2}\left(1+p^{2}\right)\left(1+q^{2}\right)},
\end{aligned}
$$

in (4.14) and upon simplification, we have the analytical solution of (5.21) as

$$
\psi(x, y, t)=L_{x}^{-1} L_{y}^{-1} L_{t}^{-1} \frac{p q-1}{s^{2}\left(1+p^{2}\right)\left(1+q^{2}\right)} .
$$

Hence the solution we get is

$$
\psi(x, y, t)=t \cos (x+y)
$$

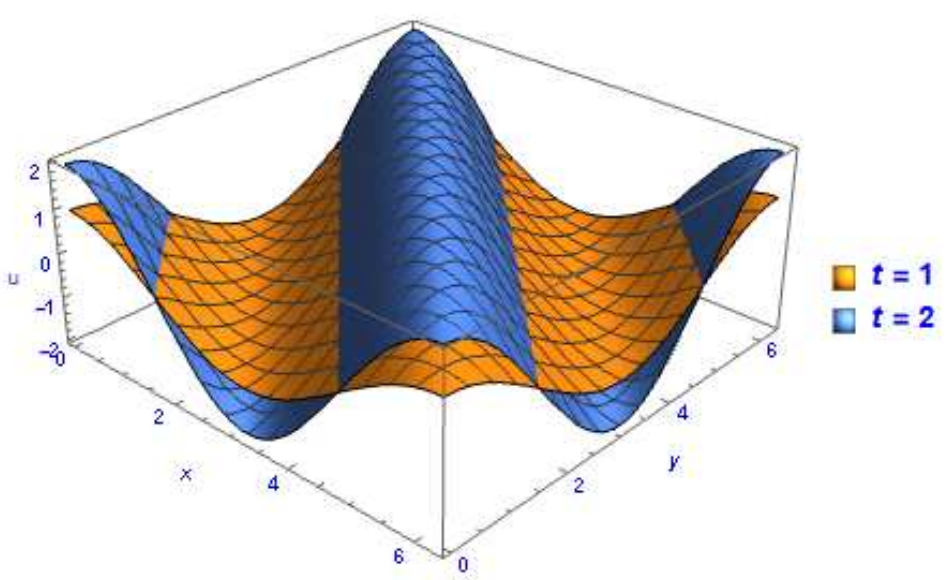

FIGURE 3. Plot for the solution $\psi(x, t)=t \cos (x+y)$ with $t=1$ and $t=2$. 


\section{CONCLUSION}

In this article, contrary to Adomian decomposition method of numerical approximation used in [10] and other numerical and analytical methods. We provided the techniques of DLT and TLT to evaluate the analytical exact solutions of PDEs problems with initial and boundary conditions. Examples are provided for the purpose of illustration via double and triple Laplace transform methods. Figures 1, 2 and 3 showed the precise graphical view of solutions of examples. Finally, we concluded that the method of LT is an easy and effective scheme for the solutions of various linear problems arising in the field of engineering. In this work, performance of LT is excellent and we recommend that its application is suitable for solution of any initial and boundary value problem of linear PDEs or ODEs.

\section{ACKNOWLEDGMENTS}

We are grateful to the anonymous reviewers for their generosity and expertise as seen by their insightful comments and constructive criticism which improved the final revised version of this article. Also we acknowledge and thank the respected editor for his useful suggestions of removing typographical/grammatical errors from the paper. Finally, we are thankful to the editor for accepting this article for publication in Punjab university journal of Mathematics.

\section{COMPETING INTEREST}

This manuscript is considered not to be of any competing interest.

\section{AUTHORS CONTRIBUTION}

All the authors contributed equally in production of this manuscript.

\section{FUNDING INFORMATION}

There is no funding source which support this manuscript financially.

\section{REFERENCES}

[1] A. Atangana, A Note on the Triple Laplace Transform and Its Applications to Some Kind of Third-Order Differential Equation, Abstr. Appl. Anal. 2013, (2013) 1-10.

[2] A. Atangana and A. Kilicman, A possible generalization of acoustic wave equation using the concept of perturbed derivative order, Math. Probl. Eng. 2013, (2013) 1-6.

[3] D. Baleanu and G. C. Wu, Some further results of the Laplace transform for variable-order fractional difference equations, Fract. Calc. Appl. Anal. 22, No.6 (2019) 1641-1654.

[4] A. Bokharia, D. Baleanu and R. Belgacem, Application of Shehu transform to Atangana-Baleanu derivatives, J. Math. Comput. Sci. 20, No.2 (2020) 101-107.

[5] R. R. Dhunde and G. L. Waghmare, Double Laplace Transform Method in Mathematical Physics, Int. J. Theor. Math. Phys. 7, No. 1 (2017) 14-20.

[6] D. G. Duffy, Transform Methods for Solving Partial Differential Equations, CRC Press, New York (2004).

[7] H. Jafri, H. Tajadodi, New method for solving a class of fractional partial differential equations with applications, Therm. Sci. 22, No. 1 (2018) 277-286.

[8] H. Jafri, H. Tajadodi, D. Baleanu, Application of a homogeneous balance method to exact solutions of nonlinear fractional evolution equations, J. Comput. Nonlinear Dynam. 9, No.2 (2014). 
[9] H. Jafari , H. Tajadodi and A. Biswas, Homotopy analysis method for solving a couple of evolution equations and comparison with Adomian's decomposition method, Waves Random Complex Media 21, No.4 (2011) 657-667.

[10] H. Jafri, H. Tajadodi and D. Baleanu, A modified variational iteration method for solving fractional Riccati differential equation by Adomian polynomials, Fract. Calc. Appl. Anal. 16, No.1 (2013) 109-122.

[11] D. Kaya, An application of the decomposition method for second order wave equations, Int. J. Comput. Math. 75, No.2 (2000) 235-245.

[12] T. Khan, K. Shah, A. Khan and R. A. Khan, Solution of fractional order heat equation via triple Laplace transform in two dimensions, Math. Methods Appl. Sci. 41, No.2 (2017) 1099-1476.

[13] Z. Korpinar, M. Inc and D. Baleanu, On the fractional model of Fokker-Planck equations with two different operator, AIMS Mathematics, 5, No.1 (2020) 236-248.

[14] H. Rehman, M. Iftikhar, S. Saleem, M. Younis and A. Mueed, A Computational Quadruple Laplace Transform for the Solution of Partial Differential Equations, Appl. Math. 5 (2014) 3372-3382.

[15] I. N. Sneddon, The use of integral transforms, Tata McGraw Hill Edition (1974). 